\title{
Manifestation, en France, du piétin-échaudage sur gazon dû à Gaeumannomyces graminis var avenae
}

\author{
P Lucas ${ }^{1}$, A Sarniguet ${ }^{1}, C$ Laurent $^{2}$ \\ ${ }^{1}$ INRA, centre de recherche de Rennes, station de pathologie végétale; \\ ${ }^{2}$ GRISP, BP 29, 35650 Le Rheu, France
}

(Reçu le 5 octobre 1991, accepté le 10 janvier 1992)

\begin{abstract}
Résumé - Des destructions de plantes en foyers circulaires ou semi-circulaires sont fréquemment observées sur parcours de golf de l'Ouest de la France. Ces dégâts affectent aussi bien la fétuque sur " fairways " que l'agrostide sur "greens". L'examen morphologique des isolats responsables de la maladie montre qu'il s'agit d'un champignon appartenant à l'espèce Gaeumannomyces graminis (Sacc) von Arx et Olivier. La longueur des ascospores produites par ces isolats, supérieure à celle d'ascospores produites par $G$ graminis var tritici Walker, permet de le rattacher à la variété avenae (Turner) Dennis. De plus, parmi 2 souches très agressives isolées l'une de blé, l'autre d'agrostide, seule celle isolée d'agrostide s'est révélée pathogène à la fois sur blé et sur avoine.
\end{abstract}

gazon / piétin-échaudage / Gaeumannomyces graminis avenae

Summary - Occurrence in France of take-all patches on turfgrass caused by Gaeumannomyces graminis var avenae. Patches of destroyed plants are often observed on turfgrass on some golf courses in western France. The attacks occur on the fairways sown with fescue as well as on greens sown with Agrostis. The morphological characteristics of the isolates responsible for the disease showed that they belong to the species Gaeumannomyces graminis (Sacc) von Arx et Olivier. The length of the ascospores $(79.3-106.8 \mu \mathrm{m})$ produced by these isolates was larger than the length of ascospores $(60.0-77.7 \mu \mathrm{m}) \mathrm{pro}-$ duced by $\mathrm{G}$ graminis var tritici Walker, leading to their identification as $\mathrm{G}$ graminis var avenae (Turner) Dennis. Furthermore, out of 2 recently isolated strains obtained 1 from wheat, the other from agrostis, only the one isolated from agrostis was pathogenic on both wheat and oat.

turfgrass / take-all patch / Gaeumannomyces graminis avenae

\section{INTRODUCTION}

Sur un terrain de golf récemment aménagé dans la région de Bénodet (Bretagne), les "greens » ensemencés en Agrostis palustris Huds cv Penncross ont présenté, en 1987, des foyers circulaires ou semi-circulaires de plantes détruites. Le centre de ces foyers est généralement recolonisé par d'autres graminées ou par des dicotylédones. L'aspect irré- gulier de la surface ainsi obtenue est alors préjudiciable, sinon à l'esthétique, du moins à l'évolution très précise de la balle, qui est recherchée dans cette partie du parcours. Sur les «fairways", ensemencés en un mélange de fétuque rouge (Festuca rubra subsp rubra L, cv Ludivine $50 \%$, cv Pernille $20 \%$ ) et d'agrostide (Agrostis tenuis Sibth, 30\%), des symptômes similaires ont pu être observés, mais avec une recolonisation centrale ultérieure du foyer due à l'un des composants du 
mélange (la fétuque), donnant à la maladie un aspect en anneaux de plantes détruites. Cette recolonisation par des plantes sensibles s'explique par le développement d'une microflore antagoniste en arrière du front de progression de la maladie (Lucas et al, 1991).

Les premiers échantillons de plantes malades reçus par le GRISP (Groupement régional d'intérêt scientifique et phytosanitaire) devaient révéler la présence, sur racines et stolons d'agrostide et sur racines et bases des tiges de fétuque, de Gaeumannomyces graminis (Sacc) von Arx et Olivier, agent du piétin-échaudage sur de nombreuses graminées et céréales. C'est sur Cynodon sp et sur Agropyron sp que Saccardo a, pour la première fois en 1875 , identifié l'agent du piétinéchaudage (Ophiobolus graminis), largement répandu dans le monde et connu depuis pour ses dégâts sur blé et sur orge. Cet agent, aujourd'hui rattaché à l'espèce Gaeumannomyces graminis, peut appartenir à 3 variétés différentes suivant sa spécificité de plante hôte et en fonction de certaines caractéristiques morphologiques (Walker, 1973). Ainsi, alors que $G$ graminis var tritici Walker (Ggt) attaque le blé, l'orge et de nombreuses graminées prairiales (Nilsson, 1969), G graminis var avenae Turner (Dennis) (Gga) est également pathogène sur avoine. Ces 2 variétés sont signalées en France, respectivement depuis 1890 par Prillieux et Delacroix en ce qui concerne Ggt et en 1967 par Lemaire, en ce qui concerne Gga. Outre leurs aptitudes parasitaires, le critère essentiel de différenciation entre ces 2 variétés repose sur la longueur respective des ascospores (Walker, 1973). Une troisième variété, $G$ graminis var graminis (Ggg), distincte des 2 précédentes par la formation à la fois d'hyphopodes simples et lobés (simples seulement pour Gga et Ggt), se développe principalement sur le riz dans les régions tropicales et subtropicales.

L'objet du présent article est de décrire les symptômes observés sur agrostide et fétuque et de rattacher à l'une ou l'autre des variétés tritici ou avenae le champignon responsable des attaques.

\section{MATÉRIEL ET MÉTHODES}

L'origine des 4 isolats comparés, 2 obtenus d'agrostide $\left(A_{1}, A_{2}\right), 2$ obtenus de blé $\left(B_{1}, B_{2}\right)$, est décrite dans le tableau 1 .

\section{Test de virulence}

Des tubes de verre $(d=22 \mathrm{~mm}, h=20 \mathrm{~cm})$ sont remplis de perlite stérile jusqu'aux $2 / 3$ de leur longueur. Deux explantats $(d=5 \mathrm{~mm})$ prélevés à la périphérie d'une culture, sur milieu PDA, d'un des isolats à tester, sont alors introduits dans le tube, avant remplissage complet de celui-ci. Après humidification de la perlite de façon que seuls les $2 \mathrm{~cm}$ inférieurs présentent un film continu d'eau, 2 caryopses de blé, cv Talent, ou d'avoine, cv Peniarth, sont semés dans chaque tube. Pour chaque isolat et chaque plante hôte, 5 répétitions sont réalisées. L'ensemble est placé sous lumière naturelle, à température ambiante du laboratoire. Chaque semaine, à partir de la $3^{e}$, le nombre de racines présentant au moins 1 nécrose, ainsi que la longueur de racine nécrosée sont appréciés par examen des plantes en place, au travers de la paroi de chaque tube.

\section{Obtention et mesure des ascospores}

Les ascospores sont obtenues par prélèvement des périthèces produits par les 4 isolats $A_{1}, A_{2}$, $B_{1}, B_{2}$, sur racines de blé, après inoculation selon la méthode décrite précédemment. Ces périthèces se forment, à la lumière, en 4-5 semaines, au laboratoire. Ils sont alors légèrement écrasés entre lame et lamelle dans une goutte d'eau, pour examen sous le microscope $(400 x)$ et mesure de la longueur des ascospores ainsi libérées.

\section{RÉSULTATS}

\section{Symptômes sur plantes}

Les plantes d'agrostide ou de fétuque attaquées présentent un système racinaire moins important que les plantes saines, mais les symptômes les plus évidents se manifestent sur les stolons d'agrostide ou à la base des

Tableau I. Origine des 4 isolats de $G$ graminis étudiés.

\begin{tabular}{clll}
\hline Origine & Plante hôte & \multicolumn{1}{c}{ Lieu } & Année \\
\hline Code isolat & & & \\
$\mathrm{A}_{1}$ & agrostide & Finistère & 1988 \\
$\mathrm{~A}_{2}$ & agrostide & Finistère & 1989 \\
$\mathrm{~B}_{1}$ & blé & Ille-et-Vilaine & 1988 \\
$\mathrm{~B}_{2}$ & blé & Loire-Atlantique & 1989 \\
\hline
\end{tabular}


tiges de fétuque. Ceux-ci jaunissent et sont ponctués de périthèces noirs caractérisés par un long col émergeant des tissus de la plante. Un examen attentif des racines (sous la loupe) révèle la présence de nécroses brunes à noirâtres, parfois peu perceptibles à l'œil nu, compte tenu de la taille de ces racines. Lorsque l'attaque devient importante, la plante entière jaunit et meurt. Les dégâts apparaissent le plus souvent au printemps et peuvent se prolonger jusqu'à l'automne pour ensuite s'atténuer pendant la période hivernale.

\section{Test de virulence}

Les longueurs de racines nécrosées sur blé ou avoine, en fonction de l'isolat utilisé, sont mesurées $35 \mathrm{j}$ après émergence des plantes (tableau II). Les 2 isolats les moins agressifs sont ceux qui ont été conservés le plus longtemps en mycothèque sur milieu synthétique (isolés en 1988) et ne sont virulents que sur blé. Des 2 isolats obtenus l'année même de l'expérience (1989), seul celui isolé d'agrostide est virulent aussi bien sur blé que sur avoine.

\section{Longueur des ascospores}

Les ascospores produites sur racines de blé par les isolats $A_{1}$ et $A_{2}$, obtenus d'agrostide, sont significativement plus longues que celles produites par les isolats $B_{1}$ et $B_{2}$, obtenus de blé (tableau III).

\section{DISCUSSION}

La mesure des longueurs d'ascospores (tableau III) permet de classer les isolats en 2 groupes, $A_{1}$ et $A_{2}$ d'une part, $B_{1}$ et $B_{2}$ d'autre part. Les différences observées dans la taille des ascospores sont conformes aux observations de Nilsson (1969), et permettent, d'une part, de rattacher les isolats $A_{1}$ et $A_{2}$ de $G$ graminis à la variété avenae et, d'autre part, de confirmer l'appartenance des 2 isolats $B_{1}$ et $B_{2}$ à la variété tritici. L'incapacité de ces 2 derniers isolats (tableau II) à attaquer l'avoine corrobore cette affirmation. En ce qui concerne les isolats $A_{1}$ et $A_{2}$, s'ils sont tous les 2 pathogènes sur blé, un seul $\left(A_{2}\right)$ provoque d'importantes nécroses sur avoine. Signalons que $A_{1}$, isolé en 1988 , soit 15 mois avant son utilisation dans cette étude, produisait à l'origine de nombreux périthèces sur milieu PDA, aptitude qu'il avait perdue par la suite. Ces modifications physiologiques de l'isolat ont pu s'accompagner de modifications du pouvoir pathogène, comme c'est souvent le cas chez $G$ graminis lorsqu'il est maintenu en culture sur milieu synthétique (Férault et al, 1979). II est d'ailleurs à noter que les 2 isolats

Tableau II. Longueur des nécroses observées 35 j après émergence des plantules de blé (cv Talent) ou d'avoine (cv Peniarth) inoculées avec des isolats de $G$ graminis obtenus d'agrostide $\left(A_{1}, A_{2}\right)$ ou de blé $\left(B_{1}, B_{2}\right)$ ou non inoculées (témoins). Moyennes de 10 répétitions. Les valeurs suivies de la même lettre ne sont pas significativement différentes pour $p=0,05$.

\begin{tabular}{lccccc}
\hline \multirow{2}{*}{ Plante } & \multicolumn{5}{c}{ Isolat } \\
\cline { 2 - 6 } & $A_{1}$ & $A_{2}$ & $B_{1}$ & $B_{2}$ & Témoin \\
\hline Blé & $6,9^{\mathrm{b}}$ & $11,5^{\mathrm{a}}$ & $7,7^{\mathrm{b}}$ & $9,3^{\mathrm{ab}}$ & $0^{\mathrm{d}}$ \\
Avoine & $1,2^{\mathrm{c}}$ & $12,8^{\mathrm{a}}$ & $0,3^{\mathrm{c}}$ & $1,3^{\mathrm{c}}$ & $0^{\mathrm{d}}$. \\
\hline
\end{tabular}

Tableau III. Longueur moyenne des ascospores issues de périthèces prélevés de racines de blé inoculé avec 2 isolats de $G$ graminis obtenus d'agrostide $\left(A_{1}, A_{2}\right)$ ou de blé $\left(B_{1}, B_{2}\right)$. Deux périthèces sont prélevés pour chaque isolat. Les valeurs suivies de la même lettre ne sont pas significativement différentes pour $p=0,05$.

\begin{tabular}{lcccc}
\hline & \multicolumn{4}{c}{ Isolat } \\
\cline { 2 - 5 } & $A_{1}$ & $A_{2}$ & $B_{1}$ & $B_{2}$ \\
\hline Longueur des ascospores $(\mu \mathrm{m})^{\star}$ & $89,7^{\mathrm{a}} \pm 10,4$ & $94,2^{\mathrm{a}} \pm 12,6$ & $71,2^{\mathrm{b}} \pm 6,5$ & $68,3^{\mathrm{b}} \pm 8,3$ \\
Nb d'ascospores mesurées & 68 & 83 & 87 & 79 \\
\hline
\end{tabular}

\footnotetext{
* Longueur \pm écart type.
} 
présentant les moindres performances pathogènes sur blé, comme sur avoine, soient $A_{1}$ et $B_{1}$ isolés en 1988, comparés à leur homologues $A_{2}$ et $B_{2}$ isolés en 1989, l'année même de l'étude.

\section{CONCLUSION}

Le piétin-échaudage des gazons peut provoquer d'importants dégâts sur parcours de golf, comme nous avons pu l'observer à Bénodet, Finistère. D'autres cas nous ont été signalés depuis, dans les Landes, le Morbihan, l'llleet-Vilaine et la Manche. II s'agit généralement de golfs récemment aménagés, mais nous avons pu observer des dégâts similaires sur des parcours de golf plus anciens, comme à Saint-Laurent près d'Auray dans le Morbihan ou à Dol-de-Bretagne en Ille-et-Vilaine.

Le champignon responsable appartient bien à l'espèce Gaeumannomyces graminis var avenae, comme le montrent les caractéristiques morphologiques et pathogéniques des souches étudiées.

L'origine des attaques reste indéterminée. Les surfaces utilisées pour les implantations des golfs que nous avons visités (Bretagne) étaient, à l'origine, des landes ou des prairies et le champignon pouvait être indigène sur des graminées spontanées. En ce qui concerne les "greens", où l'agrostide est cultivé sur substrat importé (sable ou tourbe + sable), la contamination est vraisemblablement secondaire et a pu se réaliser par transport d'inoculum lors des fréquentes opérations culturales (tonte, aération des sols, etc) ou par les joueurs eux-mêmes (chaussures à pointes). Enfin, contrairement au piétin-échaudage sur céréales où les périthèces ne se forment qu'à la fin du cycle végétatif des plantes, dans le cas présent, les ascospores peuvent jouer un rôle dans la dissémination de la maladie comme semblent le montrer certaines expérimentations réalisées en Grande-Bretagne (Baldiwin et al, 1991).

Des études sont poursuivies, visant à la mise au point de méthodes de lutte contre ce parasite.

\section{RÉFÉRENCES}

Baldwin NA, Capper AL, Yarham DJ (1991) Evaluation of biological agents for the control of take-all patch (Gaeumannomyces graminis) of fine turf. In: Biotic interactions and soil-borne diseases. (ABR Beemster, GJ Bollen, M Gerlagh, MA Ruissen, B Schippers, A Tempel eds). Elsevier, Amsterdam, 231-235

Férault AC, Tivoli B, Lemaire JM, Spire D (1979) Étude de l'évolution comparée du niveau d'agressivité et du contenu en particules de type viral d'une souche de Gaeumannomyces graminis (Sacc) Arx et Olivier (Ophiobolus graminis Sacc). Ann Phytopathol 11, 185-191

Lemaire JM (1967) Note sur l'apparition en France de l'Ophiobolus graminis var avenae EM Turner. CR Séances Acad Agric Fr 18 avril 1967, 480484

Lucas P, Sarniguet A, Lelarge S (1991) Bacterial populations related to the progress of the disease in take-all patches on turf grass. In: Biotic interactions and soil-borne diseases. (ABR Beemster, GF Bollen, M Gerlagh, MA Ruissen, B Schippers, A Tempel, eds). Elsevier, Amsterdam, 264-270

Nilsson HE (1969) Studies of root and foot rot diseases of cereals and grasses. I. On resistance to Ophiobolus graminis Sacc Lantbrukshögsk Ann 35, 275-807

Prillieux EE, Delacroix G (1890) La maladie du pied du blé, causée par l'Ophiobolus graminis Sacc Bull Soc Mycol Fr 6, 110-113

Saccardo PA (1875) Fungi veneti novi vel critici. Ser. II. Nuovo G Bot Ital 7, 299-329

Walker J (1973) Gaeumannomyces graminis var graminis, var avenae and var tritici. CMI Description of Pathogenic Fungi and Bacteria, Nos 381,382 and 383 